\title{
Asymptotic reflection of linear water waves by submerged horizontal porous plates
}

\author{
David V. Evans · Malte A. Peter
}

\begin{abstract}
On the basis of linear water-wave theory, an explicit expression is presented for the reflection coefficient $R^{\infty}$ when a plane wave is obliquely incident upon a semiinfinite porous plate in water of finite depth. The expression, which correctly models the singularity in velocity at the edge of the plate, does not rely on knowledge of any of the complex-valued eigenvalues or corresponding vertical eigenfunctions in the region occupied by the plate. The solution $R^{\infty}$ is the asymptotic limit of the reflection coefficient $R$ as $a \rightarrow \infty$, for a plate of finite length $a$ bounded by a rigid vertical wall, and forms the basis of a rapidly convergent expansion for $R$ over a wide range of values of $a$. The special case of normal incidence is relevant to the design of submerged wave absorbers in a narrow wave tank. Modifications necessary to account for a finite submerged porous plate in a fluid extending to infinity in both horizontal directions are discussed.
\end{abstract}

Keywords Water waves porous plate $\cdot$ Wiener-Hopf method $\cdot$ residue calculus method

\section{Introduction}

The interaction of waves with porous structures such as rubble-mound breakwaters has long been of interest to coastal engineers as a method of reducing wave transmission and number of theoretical research papers have addressed the problem. A good review of the literature is provided by [1].

In this paper, we shall use classical linear water-wave theory to consider the scattering of an incident wave-train by a submerged horizontal porous plate in water of

MAP was supported by Marsden grant UOO308 from the New Zealand government.

David V. Evans

School of Mathematics, University of Bristol, United Kingdom

E-mail: d.v.evans@bristol.ac.uk

Malte A. Peter

Department of Mathematics, University of Auckland, New Zealand

Present address: Institute of Mathematics, University of Augsburg, Germany and

Augsburg Centre for Innovative Technologies, University of Augsburg, Germany

E-mail: malte.peter@math.uni-augsburg.de 
finite depth. We have in mind the application to a two-dimensional wave flume where the waves produced by the wavemaker at one end are required to be absorbed at the other so that reflections do not interfere with the modelling of beam sea scattering by a long body in an infinite ocean. This is usually achieved by installing a sloping beach or a region near the end of the flume consisting of a dense mesh of absorbing material.

Our theoretical formulation is based on the work of $\mathrm{Wu}$ et al. [1], who considered the reflection by a vertical wall terminating the submerged finite length porous plate, which in turn is based on Chwang \& Wu [2], who considered the scattering by a submerged porous circular disk. We adopt their model of the porous plate in which the boundary condition to be satisfied on the plate is that the normal velocities of the fluid above and below the plate are continuous and proportional to the jump in pressure across the plate. The approach of Chwang \& Wu [2] and Wu et al. [1], which is typical of many other papers (see, for example, [3] for the submerged elastic plate), is to construct eigenfunction expansions in the water region, $x<0$, and in the plate region, $0<x<a$ and match the potential and horizontal velocities across the interface $x=0$. By using orthogonality relations, they derived two coupled infinite systems of equations for the unknown expansion coefficients in each region, which they then computed by truncation of the systems.

Here, we proceed differently for a number of reasons. We begin by considering the situation where the plate is of infinite extent so that the powerful Wiener-Hopf method can be used to derive an exact solution. We generalise the solution to include obliquely incident waves from $x<0$ approaching the plate which occupies $x>0,-\infty<y<$ $\infty, z=-d$, which in fact expedites the solution. In addition to the obvious advantage of having an exact solution to the problem, there are other more important reasons for using the Wiener-Hopf method. One of these is the fact that the method builds in to the solution the required square-root singularity of the fluid velocity at the edge of the submerged plate which is overlooked in the formulation of [1]. This omission leads to slowly convergent expansion coefficients casting doubt on the accuracy of numerical computations. For example, Porter and Evans [4, p. 156] report that use of the eigenfunction matching method to determine the scattering by a fixed partially immersed vertical barrier requires the solution of a 400-by-400 system of equations in order to achieve two-figure accuracy. This problem can be overcome in the matched eigenfunction-expansion approach by using the so-called residue calculus method where the singularity is again built in to the form of the solution, see, for example, [5, pp. 148166]. Assuming that the roots of the complex dispersion relation produced by the plate are all simple, this technique leads to an analytic expression for the reflection coefficient $R^{\infty}$, which is identical to the one obtained by the Wiener-Hopf method together with an infinite product factorisation.

However, there is a more fundamental difficulty with all matched eigenfunctionexpansion methods for porous-plate problems, which has been highlighted by McIver [6]. Because of the complicated boundary condition to be satisfied on the plate, the complex dispersion equation arising leads to an eigenvalue problem, which is not selfadjoint, so that standard Sturm-Liouville theory does not apply. McIver shows how this can be overcome in a simpler related problem but it requires knowledge of which (and when) the eigenvalues are multiple rather than simple zeroes of the dispersion relation, which have more complicated eigenfunctions. Even if it is assumed that all eigenvalues are simple, which is not true in general, it is not easy to track these complex zeroes, particularly if a large number of them is required. 
These problems are overcome by using the Wiener-Hopf approach, which avoids needing to know the roots in the plate region. Using a Cauchy integral method, we show that the expression for the reflection coefficient $R^{\infty}$ can be expressed explicitly in terms of the known eigenvalues in $x<0$ and an easily computed integral, which does not require any knowledge of the eigenfunctions in $x>0$.

Solutions to other water-wave interaction problems involving horizontal plates using the Wiener-Hopf method or the residue calculus technique can be found in the literature. These include the submerged fixed rigid plate (commonly referred to as a dock) $[7,8]$, [5, pp. 151-156] as a structure leading to a simple boundary condition but including submergence and the elastic plate at the water surface $[9,10,11,12,13]$ as a structure implying a more complicated boundary condition but without submergence. The problem considered here involves both complications: submergence and a nontrivial boundary condition. In particular, the porous-plate boundary condition leads to the difficulties mentioned above, which are not present in the problems involving docks or elastic plates.

We next consider the finite-plate problem, where the plate is backed by a wall, which is exactly the problem considered by $\mathrm{Wu}$ et al. [1]. While the Wiener-Hopf method does not work for this problem owing to the semi-infinite extent of the domain, we show how the classical eigenfunction method can be improved by using the residue calculus technique, which provides a powerful rapidly convergent solution which again builds in the necessary singularity. We also show how the method can be easily adapted to solve for a porous plate of finite length in a fluid of infinite extent in both horizontal directions.

Numerical computations confirm the validity of our approach and illuminate the slow convergence of the standard eigenfunction matching method for both the semiinfinite plate and the finite plate backed by a wall.

The paper is organised as follows: The governing equations are discussed in Sect. 2 and the solution to the semi-infinite plate problem by the Wiener-Hopf method is derived in Sect. 3. In Sect. 4, the finite-plate problem is solved using the residue calculus technique. Some numerical comparisons of the methods are given in Sect. 5 and conlusions are formulated in Sect. 6 . The paper has four appendices containing the splitting of the Wiener-Hopf function $K(s)$ by the Cauchy integral method (Appendix A), some technical details related to the simplification of the expression for the reflection coefficient (Appendix B), the splitting of the Wiener-Hopf function $K(s)$ by a standard infinite-product expansion (Appendix $\mathrm{C}$ ) as well as a brief discussion of the special case of a plate with porosity zero, i.e. a dock, (Appendix D).

A preliminary summary of this paper has recently appeared in [14].

\section{Governing equations for the semi-infinite submerged porous plate}

On the basis of linear water-wave theory there exists a harmonic velocity potential whose dependence on $y$ and $t$ is assumed to be proportional to $\mathrm{e}^{\mathrm{i}(\beta y-\omega t)}$ to allow for obliquely incident waves of frequency $\omega / 2 \pi$, where $\beta$ is the component of the wavenumber in the $y$-direction. Then, the reduced (complex) potential is $\phi(x, z)$ and the full potential is $\operatorname{Re}\left[\phi(x, z) \mathrm{e}^{\mathrm{i}(\beta y-\omega t)}\right]$. The problem is now two-dimensional and the free water surface is chosen to be $\{(x, z) \mid z=0\}$ and the porous plate occupies $\Gamma^{\mathrm{p}}=\{(x, z) \mid x>0, z=-d\}$. The water domain is $\left\{(x, z) \in \mathbb{R}^{2} \mid-h<z<0\right\} \backslash \bar{\Gamma}^{\mathrm{p}}$, which we denote by $\Omega$ for brevity. 
The mathematical description of the time-harmonic problem is as follows (cf. [5, Chap. 1] for a general introduction). It is assumed that a plane wave, making an angle $\theta_{\text {inc }}$ with the $x$-axis, is incident on the submerged plate from $x=-\infty$. Then the reduced complex potential $\phi$ satisfies

$$
\begin{aligned}
-\Delta \phi+\beta^{2} \phi & =0, & & \text { in } \Omega, \\
\partial_{z} \phi & =0, & & \text { on } z=-h, \\
\partial_{z} \phi & =\alpha \phi, & & \text { on } z=0, \\
\left.\partial_{z} \phi\right|_{z=-d_{-}} & =\left.\partial_{z} \phi\right|_{z=-d_{+}}=\mathrm{i} \mu[\phi], & & \text { on } \Gamma^{\mathrm{p}}, \\
|\nabla \phi| & =\mathcal{O}\left(r^{-1 / 2}\right) & & \text { as } r=\left(x^{2}+(z+d)^{2}\right)^{1 / 2} \rightarrow 0,
\end{aligned}
$$

where $\alpha=\omega^{2} / g$ and $g$ is gravitational acceleration. Equation (1d) is derived from the assumption that the normal velocity of the fluid passing through the porous plate is proportional to the pressure difference across it. For a fuller discussion of this see [15]. Here $\mu=\sigma \omega$ for sake of brevity, where $\sigma$ is related to the properties of the porous plate and $\operatorname{Re} \sigma>0$, and $[\phi]$ denotes the jump of $\phi$ across $\Gamma^{\mathrm{p}}:[\phi]=\left.\phi\right|_{z=-d_{-}}-\left.\phi\right|_{z=-d_{+}}$. Equation (1e) reflects the infinite speed of the fluid around the sharp edge of the plate (see [5, p. 40], for a discussion). Finally, radiation conditions are needed appropriate to the scattering of the incident wave. In what follows, we will write $c=h-d$ for brevity.

Consider in $x<0$ the expression $\mathrm{e}^{ \pm \kappa_{n} x} \psi_{n}(z)$ where

$$
\psi_{n}(z)=\frac{\cos k_{n}(z+h)}{\cos k_{n} h}, \quad n=0,1,2, \ldots,
$$

$\kappa_{n}=\left(k_{n}^{2}+\beta^{2}\right)^{1 / 2}$ and where the numbers $k_{n}, n \geq 1$, are the positive real roots of the relation

$$
\alpha+k_{n} \tan k_{n} h=0
$$

and $k_{0}=-\mathrm{i} k$ is the single negative imaginary root of (3). The positive real wavenumber $k$ is thus related to $\alpha$ by the relation $\alpha=k \tanh k h$. Then, $\mathrm{e}^{ \pm \kappa_{n} x} \psi_{n}(z)$ satisfies (1a), (1b) and (1c). Thus, a wave of unit potential amplitude, obliquely incident from $x=$ $-\infty$ on the submerged plate, has the form $\mathrm{e}^{\mathrm{i} \kappa x} \psi_{0}(z)$ where $\kappa \equiv \mathrm{i} \kappa_{0}=k \cos \theta_{\text {inc }}$ and $\beta=k \sin \theta_{\text {inc. }}$. We can now complete the conditions on $\phi$ by demanding

$$
\phi \sim\left(\mathrm{e}^{\mathrm{i} \kappa x}+R^{\infty} \mathrm{e}^{-\mathrm{i} \kappa x}\right) \psi_{0}(z) \text { as } x \rightarrow-\infty .
$$

For the region $x>0$, we assume the effect of the plate is to reduce progressively the wave amplitude along its length. Thus we prescribe

$$
\phi \rightarrow 0 \text { as } x \rightarrow \infty \text {. }
$$

\section{The Wiener-Hopf solution}

In this section, the problem with the semi-infinite plate as introduced in the previous section is solved using the Wiener-Hopf method. The general procedure is similar to that for a submerged dock, which, in particular, follows as a special case (also cf. Appendix D). The Wiener-Hopf splitting function $K(s)$ is factorised using the Cauchy integral method, thereby yielding an expression for the reflection coefficient, which does not involve the roots of the denominator of $K(s)$. Thus, it is not required to 
calculate the roots of a dispersion relation for the plate region. In general, this method is useful for any problem where the roots of the dispersion relation are difficult to compute and where only the reflection coefficient is of interest.

We split the potential $\phi$ as follows:

$$
\phi(x, z)=\psi_{0}(z) \mathrm{e}^{\mathrm{i} \kappa x}+ \begin{cases}\phi_{1}(x, z), & -d<z<0 \\ \phi_{2}(x, z), & -h<z<-d .\end{cases}
$$

Thus, $\phi_{1}$ and $\phi_{2}$ have to satisfy (1a), (1b), (1c), (1e) and also

$$
\begin{aligned}
\partial_{z} \phi_{1} & =\partial_{z} \phi_{2}, & & \text { on } z=-d, \\
\phi_{1} & =\phi_{2}, & & \text { on } z=-d, x<0, \\
\partial_{z} \phi_{j} & =\mathrm{i} \mu\left(\phi_{2}-\phi_{1}\right)-\tilde{\alpha} \mathrm{e}^{i \kappa x}, & & \text { on } \Gamma^{\mathrm{p}}, \\
\phi_{j} & \sim R^{\infty} \mathrm{e}^{-\mathrm{i} \kappa x} \psi_{0}(z), & & \text { as } x \rightarrow-\infty, \\
\phi_{j} & +\mathrm{e}^{\mathrm{i} \kappa x} \psi_{0}(z) \rightarrow 0, & & \text { as } x \rightarrow+\infty,
\end{aligned}
$$

and $j=1,2$, where $\tilde{\alpha}=k \sinh k c / \cosh k h$. We let $\Phi$ be the Fourier transform of $\phi$ with respect to $x$,

$$
\Phi(s, z)=\int_{-\infty}^{\infty} \phi(x, z) \mathrm{e}^{\mathrm{i} s x} \mathrm{~d} x,
$$

and $\Phi^{-}$and $\Phi^{+}$the negative and positive half-range Fourier transforms of $\phi$, respectively,

$$
\Phi^{-}(s, z)=\int_{-\infty}^{0} \phi(x, z) \mathrm{e}^{\mathrm{i} s x} \mathrm{~d} x, \quad \Phi^{+}(s, z)=\int_{0}^{\infty} \phi(x, z) \mathrm{e}^{\mathrm{i} s x} \mathrm{~d} x .
$$

Then, $\Phi=\Phi^{-}+\Phi^{+}$and $\Phi^{+}$is regular in the upper complex plane $\mathcal{D}^{+}=\{s \in \mathbb{C} \mid \operatorname{Im}(s+$ $\kappa)>0\}$ while $\Phi^{-}$is regular in the lower complex plane $\mathcal{D}^{-}=\{s \in \mathbb{C} \mid \operatorname{Im}(\kappa-s)>0\}$. To ensure convergence of the transforms, we shall assume that $\kappa$ has a small imaginary part $\varepsilon$ and we will let $\varepsilon \rightarrow 0$ finally. Then,

$$
\mathcal{D}^{+}=\{s \in \mathbb{C} \mid \operatorname{Im} s>-\varepsilon\}, \quad \mathcal{D}^{-}=\{s \in \mathbb{C} \mid \operatorname{Im} s<\varepsilon\}
$$

and we define $\mathcal{D}=\mathcal{D}^{+} \cap \mathcal{D}^{-}=\{s \in \mathbb{C}|| \operatorname{Im} s \mid<\varepsilon\}$.

The transformed version of (1a) is

$$
\partial_{z z} \Phi-\gamma^{2} \Phi=0, \quad s \in \mathcal{D}, \quad \text { where } \gamma^{2}=s^{2}+\beta^{2} .
$$

Then,

$$
\Phi_{1}(s, z)=A(s)(\gamma \cosh \gamma z+\alpha \sinh \gamma z)
$$

satisfies (1a) and (1c), where $\gamma=\left(s^{2}+\beta^{2}\right)^{1 / 2}$, and

$$
\Phi_{2}(s, z)=B(s) \cosh \gamma(z+h)
$$

satisfies (1a) and (1b). Transformation of (7a) for $x>0$ and (7c) gives

$$
\partial_{z} \Phi_{1}^{+}(s,-d)=\partial_{z} \Phi_{2}^{+}(s,-d)=\mathrm{i} \mu\left(\Phi_{2}^{+}(s,-d)-\Phi_{1}^{+}(s,-d)\right)-\mathrm{i} \tilde{\alpha} /(s+\kappa) .
$$

Equations (7a) for $x<0$ and $(7 \mathrm{~b})$ respectively become

$$
\begin{aligned}
\partial_{z} \Phi_{1}^{-}(s,-d) & =\partial_{z} \Phi_{2}^{-}(s,-d) \equiv Q^{-}(s), \\
\Phi_{1}^{-}(s,-d) & =\Phi_{2}^{-}(s,-d) .
\end{aligned}
$$


Differentiating (12) and (13) with respect to $z$ and putting $z=-d$, we obtain

$$
\begin{aligned}
& \partial_{z} \Phi_{1}(s,-d)=\gamma A(s)(\alpha \cosh \gamma d-\gamma \sinh \gamma d), \\
& \partial_{z} \Phi_{2}(s,-d)=\gamma B(s) \sinh \gamma c
\end{aligned}
$$

where $c=h-d$. Addition of (14) and (15) and comparison with (17) and (18) gives

$$
A(s)=\frac{Q^{-}(s)+\mathrm{i} \mu D^{+}(s)-\mathrm{i} \tilde{\alpha} /(s+\kappa)}{\gamma(\alpha \cosh \gamma d-\gamma \sinh \gamma d)}, \quad B(s)=\frac{Q^{-}(s)+\mathrm{i} \mu D^{+}(s)-\mathrm{i} \tilde{\alpha} /(s+\kappa)}{\gamma \sinh \gamma c},
$$

where

$$
\begin{aligned}
D^{+}(s) & =\Phi_{2}^{+}(s,-d)-\Phi_{1}^{+}(s,-d) \\
& =\Phi_{2}^{+}(s,-d)-\Phi_{1}^{+}(s,-d)+\Phi_{2}^{-}(s,-d)-\Phi_{1}^{-}(s,-d) \\
& =\Phi_{2}(s,-d)-\Phi_{1}(s,-d) \\
& =B(s) \cosh \gamma c-A(s)(\gamma \cosh \gamma d-\alpha \sinh \gamma d),
\end{aligned}
$$

where we have made use of (16). Substituting (19) into (20), we obtain

$$
\begin{aligned}
D^{+}(s) & =\left(Q^{-}(s)+\mathrm{i} \mu D^{+}(s)-\mathrm{i} \tilde{\alpha} /(s+\kappa)\right)\left(\frac{\cosh \gamma c}{\gamma \sinh \gamma c}-\frac{\gamma \cosh \gamma d-\alpha \sinh \gamma d}{\gamma(\alpha \cosh \gamma d-\gamma \sinh \gamma d)}\right) \\
& =\left(Q^{-}(s)+\mathrm{i} \mu D^{+}(s)-\mathrm{i} \tilde{\alpha} /(s+\kappa)\right) \frac{\gamma \sinh \gamma h-\alpha \cosh \gamma h}{\gamma \sinh \gamma c(\gamma \sinh \gamma d-\alpha \cosh \gamma d)}
\end{aligned}
$$

or, equivalently,

$$
D^{+}+\left(Q^{-}(s)-\mathrm{i} \tilde{\alpha} /(s+\kappa)\right) K(s)=0,
$$

where

$$
K(s)=\frac{\gamma \sinh \gamma h-\alpha \cosh \gamma h}{\gamma \sinh \gamma c(\gamma \sinh \gamma d-\alpha \cosh \gamma d)-\mathrm{i} \mu(\gamma \sinh \gamma h-\alpha \cosh \gamma h)}
$$

is the Wiener-Hopf splitting function. Now it is shown in Appendix A that we can write

$$
K(s)=K_{+}(s) K_{-}(s),
$$

where $K_{ \pm}(s)$ is non-zero and regular in $\mathcal{D}_{ \pm}$. Thus, $(22)$ can be written as

$$
\frac{D^{+}(s)}{K_{+}(s)}-\frac{\mathrm{i} \tilde{\alpha}}{s+\kappa} K_{-}(-\kappa)=-Q^{-}(s) K_{-}(s)+\frac{\mathrm{i} \tilde{\alpha}}{s+\kappa}\left(K_{-}(s)-K_{-}(-\kappa)\right),
$$

where the left (right)-hand side of $(25)$ is regular in $\mathcal{D}_{+}\left(\mathcal{D}_{-}\right)$. Now (1e) implies that $\Phi_{ \pm}^{\prime}(s,-d)=\mathcal{O}\left(s^{-1 / 2}\right)$ as $s \rightarrow \infty$ in $\mathcal{D}_{ \pm}$. It follows from (12) and (13) that the same is true of $Q^{-}(s)$ and $D^{+}(s)$. Together with the result $K_{ \pm}(s)=\mathcal{O}\left(s^{-1 / 2}\right)$ as $s \rightarrow \infty$ in $\mathcal{D}_{ \pm}$proved in Appendix A, it follows from (24) that either side of (25) vanishes for large $|s|$ so that by Liouville's theorem each side must be identically zero. Thus, using (12), (19), (21) we finally obtain, from (25),

$$
\begin{aligned}
\Phi_{1}(s, z) & =D^{+}(s) \frac{\sinh \gamma c(\gamma \cosh \gamma z+\alpha \sinh \gamma z)}{\alpha \cosh \gamma h-\gamma \sinh \gamma h} \\
& =\frac{\mathrm{i} \tilde{\alpha} K_{-}(-\kappa) K_{+}(s)}{s+\kappa} \frac{(\gamma \cosh \gamma z+\alpha \sinh \gamma z) \sinh \gamma c}{\alpha \cosh \gamma h-\gamma \sinh \gamma h} .
\end{aligned}
$$


Similarly, using (12), (19), (21),

$$
\Phi_{2}(s, z)=\frac{\mathrm{i} \tilde{\alpha} K_{-}(-\kappa) K_{+}(s)}{s+\kappa} \frac{(\alpha \cosh \gamma d-\gamma \sinh \gamma d) \cosh \gamma(z+h)}{\alpha \cosh \gamma h-\gamma \sinh \gamma h}
$$

and from the inversion formula,

$$
\phi_{j}(x, z)=\frac{1}{2 \pi} \int_{\mathcal{C}} \mathrm{e}^{-\mathrm{i} s x} \Phi_{j}(s, z) \mathrm{d} s, \quad j=1,2,
$$

where the contour $\mathcal{C}$ passes from $-\infty$ to $+\infty$ in $\mathcal{D}$.

For $x<0$, we deform $\mathcal{C}$ upwards into $\mathcal{D}_{+}$, picking up the contributions from the poles of the integrand at $s=\mathrm{i} \kappa_{n}, \gamma=\mathrm{i} k_{n}, n \geq 0$. The reflected wave arises from the simple pole at $s=\kappa, \gamma=k$, where $n=0$.

We find that

$\phi_{j}(x, z)=R^{\infty} \psi_{0}(z) \mathrm{e}^{-\mathrm{i} \kappa x}-2 \mathrm{i} \tilde{\alpha} K_{-}(-\kappa) \sum_{n=1}^{\infty} \frac{k_{n}^{2} \sin k_{n} c \cos k_{n} h}{\kappa_{n}\left(2 k_{n} h+\sin 2 k_{n} h\right)} K_{+}\left(\mathrm{i} \kappa_{n}\right) \psi_{n}(z) \mathrm{e}^{\kappa_{n} x}$,

where

$$
R^{\infty}=\frac{k^{3} \sinh ^{2} k c K_{+}(\kappa) K_{-}(-\kappa)}{\kappa^{2}(2 k h+\sinh 2 k h)} .
$$

Notice that the expression is the same for $j=1$ and $j=2$ as expected since the potentials and their $x$-derivatives are continuous across $z=-d$ for $x<0$.

This expression for $R^{\infty}$ can be simplified considerably making use of results in Appendix A and Appendix B. It turns out that

$$
R^{\infty}=-\exp (-2 \mathrm{i} \Theta(\kappa))
$$

where

$\Theta(\kappa)=I(\kappa)+2 \arctan (\beta / \kappa)+\chi(\kappa)+\sum_{n=1}^{\infty}\left(\arctan \left(\kappa / \kappa_{n}\right)-\arctan \left(\kappa / c_{n}^{\prime}\right)-\arctan \left(\kappa / d_{n}^{\prime}\right)\right)$,

where $c_{n}=n \pi / c, d_{n}=n \pi / d$ and

$$
\kappa_{n}=\left(k_{n}^{2}+\beta^{2}\right)^{1 / 2}, c_{n}^{\prime}=\left(c_{n}^{2}+\beta^{2}\right)^{1 / 2}, d_{n}^{\prime}=\left(d_{n}^{2}+\beta^{2}\right)^{1 / 2}
$$

and $\chi$ is defined in (99) while $I$ is given by (103).

It is of interest to determine $R^{\infty}$ for values of the physical parameters at which the zeroes of the denominator of $K(s)$ are all simple. This allows us to make a comparison with the Cauchy integral method, which makes no such assumption. Thus, we assume that the zeros of the denominator of $K(s)$ are of the form $\gamma=\gamma_{n}= \pm \mathrm{i} l_{n}$, or $s=$ $\pm \mathrm{i} \lambda_{n}, n=0,1,2, \ldots$, where the $l_{n}$ are all complex. Care must be taken in the numbering of the roots, which we also denote as $\lambda_{n}^{ \pm}, n=1,2, \ldots$, as they arise as perturbations of the roots above (below) the plate in the limit case of an impermeable dock $(\sigma=0)$, also cf. Appendix D. Keeping in mind that, from Appendix A, $F(s)=K^{\mathrm{O}}(s) / K^{\mathrm{d}}(s)$, where $K^{\mathrm{o}}$ is defined by $(95)$ and $K^{\mathrm{d}}$ is the denominator of $K$, we may then write

$$
\begin{aligned}
F(s) & =\frac{\gamma^{2} \sinh \gamma c \sinh \gamma d}{(-\mathrm{i} \mu \alpha) \prod_{n=0}^{\infty}\left(1+\gamma^{2} / l_{n}^{2}\right)} \\
& =\frac{\left.\left(s^{2}+\beta^{2}\right)^{2} c d \prod_{n=1}^{\infty}\left(\left(c_{n}^{\prime 2}+s^{2}\right) / c_{n}^{2}\right)\left(d_{n}^{\prime 2}+s^{2}\right) / d_{n}^{2}\right)}{(-\mathrm{i} \mu \alpha) \prod_{n=1}^{\infty}\left(\left(\lambda_{n}^{+2}+s^{2}\right) / l_{n}^{+2}\right)\left(\left(\lambda_{n}^{-2}+s^{2}\right) / l_{n}^{-2}\right)}
\end{aligned}
$$


and the infinite product in the denominator converges because of the behaviour of the $l_{n}$ as $n \rightarrow \infty$. We need to evaluate

$$
I(\kappa)=\frac{1}{\pi} \int_{0}^{\infty} \frac{\log F(\kappa t)}{t^{2}-1} \mathrm{~d} t
$$

We make use of the result

$$
\frac{1}{\pi} \int_{0}^{\infty} \frac{\log \left(a^{2}+b^{2} t^{2}\right)}{t^{2}-1} \mathrm{~d} t=\arctan (b / a)
$$

[see 16, Sect. 4.295, eq. (8)] and we find from (32), (33) that

$$
\begin{aligned}
I(\kappa)= & 2 \arctan (\kappa / \beta) \\
& +\sum_{n=1}^{\infty}\left(\arctan \left(\kappa / d_{n}^{\prime}\right)-\arctan \left(\kappa / \lambda_{n}^{+}\right)+\arctan \left(\kappa / c_{n}^{\prime}\right)-\arctan \left(\kappa / \lambda_{n}^{-}\right)\right) .
\end{aligned}
$$

Thus, combining (30) and (35), we obtain

$$
\Theta(\kappa)=\pi+\chi(\kappa)+\sum_{n=1}^{\infty}\left(\arctan \left(\kappa / \kappa_{n}\right)-\arctan \left(\kappa / \lambda_{n}^{+}\right)-\arctan \left(\kappa / \lambda_{n}^{-}\right)\right)
$$

The same expression can also be obtained directly using a standard infinite-product decomposition to split $K(s)$. The details are given in Appendix C.

\section{The finite submerged porous plate}

We consider the problem of the submerged porous plate as before but with a rigid vertical wall at $x=a>0$. This problem corresponds to waves being symmetric about $x=a$ and is precisely the problem considered in $[1,17]$.

Because of the semi-infiniteness of the domain, the Wiener-Hopf method can no longer be employed. Instead, under the assumption of simple roots of the dispersion relations, we show how to use the residue calculus technique to convert the problem to an infinite system of equations for quickly decaying coefficients. Progress is also possible if there are non-simple roots but this requires the precise knowledge of these roots and the eigenfunction expansions are different (see [6] on this point for a related problem).

The Wiener-Hopf method and the residue calculus technique are closely related. However, the Wiener-Hopf method works for problems in a halfplane as well as a strip, which is not the case for the residue calculus method. To show the equivalence for strip problems is not easy, although Mittra and Lee [18, pp. 150-153] showed that the methods are precisely equivalent in a related simpler problem.

The resulting expression for the reflection coefficient for the finite plate is actually a multiplicative correction of the semi-infinite-plate reflection coefficient $R^{\infty}$. The correction term is given in terms of a rapidly convergent series expansion. It is noteworthy that the residue calculus technique also yields an exact expression for $R^{\infty}$ as is shown below. Furthermore, it is discussed at the end of this section, how to modify the equations to account for a finite porous plate in a fluid extending to infinity in both positive and negative $x$-direction. 
4.1 Governing equations and eigenfunction expansions

The plate is again denoted by $\Gamma^{\mathrm{p}}=\{(x, z) \mid 0<x<a, z=-d\}$ and the domain occupied by water is now $\Omega=\{(x, z) \mid-\infty<x<a,-h<z<0\} \backslash \bar{\Gamma}^{\mathrm{p}}$. Using the same notation as in the previous sections, the system of equations to be satisfied by the time-harmonic water velocity potential is given by system (1) together with the radiation condition (4), where $R^{\infty}$ is replaced by the finite-plate reflection coefficient $R$, and the homogeneous Neumann condition at the vertical wall

$$
\partial_{x} \phi=0 \text { at } x=a .
$$

For the finite plate, we split the potential into $\phi^{-}$(for $\left.x<0\right)$ and $\phi^{+}$(for $0<x<$ a). From the discussion in Sect. 2, it is easy to see that

$$
\phi^{-}(x, z)=\psi_{0}(z) \mathrm{e}^{\mathrm{i} \kappa x}+\sum_{m=0}^{\infty} a_{m} \psi_{m}(z) \mathrm{e}^{\kappa_{m} z}
$$

where $\psi_{n}$ is defined in (2), satisfies the required conditions for $x<0$.

From (1d), we see that the potential $\phi^{+}$is discontinuous at $z=-d$ but its $z$ derivative is continuous. Suitable vertical eigenfunctions $\Psi_{m}(z)$ satisfying (1b) and (1c) are given by

$$
\Psi_{m}(z)= \begin{cases}\bar{\Psi}_{m}(z)=p_{m}\left(l_{m} \cos l_{m} z+\alpha \sin l_{m} z\right), & -d<z<0, \\ \underline{\Psi}_{m}(z)=q_{m} \cos l_{m}(z+h), & -h<z<-d,\end{cases}
$$

where the separation constants $l_{m}$ must be the same above and below the plate because of (1d). In order to satisfy the first equality in (1d),

$$
\bar{\Psi}_{m}^{\prime}(-d)=\underline{\Psi}_{m}^{\prime}(-d)
$$

i.e.

$$
p_{m}\left(l_{m} \sin l_{m} d+\alpha \cos l_{m} d\right)+q_{m} \sin l_{m} c=0
$$

must be satisfied. This determines $q_{m}$ in terms of $p_{m}$. From the second equality in (1d), we get

$$
p_{m}\left(l_{m}^{2} \sin l_{m} d+\alpha l_{m} \cos l_{m} d\right)+\mathrm{i} \mu p_{m}\left(l_{m} \cos l_{m} d-\alpha \sin l_{m} d\right)=\mathrm{i} \mu q_{m} \cos l_{m} c .
$$

Using (41), this can be reformulated as

$$
\frac{l_{m}}{\mathrm{i} \mu}+\frac{l_{m} \sin l_{m} h+\alpha \cos l_{m} h}{\left(l_{m} \sin l_{m} d+\alpha \cos l_{m} d\right) \sin l_{m} c}=0 .
$$

This is the dispersion relation for the submerged plate. Its roots in the fourth quadrant of the complex plane $l_{m}, m=0,1, \ldots$, are numbered with increasing real part. As before, we write $\lambda_{m}=\left(l_{m}^{2}+\beta^{2}\right)^{1 / 2}$. Note that (43) is equivalent to $K^{\mathrm{d}}\left( \pm \mathrm{i} \lambda_{m}\right)=0$, $m=0,1, \ldots$

We still need one more condition to specify $p_{m}$. We normalise $\Psi_{m}(z)$ such that

$$
\int_{-h}^{0} \Psi_{m}(z) \Psi_{n}(z) \mathrm{d} z=\delta_{m n}
$$


i.e.

$$
\begin{aligned}
1 & =\int_{-h}^{0} \Psi_{m}^{2}(z) \mathrm{d} z=q_{m}^{2} \int_{-h}^{-d} \cos ^{2} l_{m}(z+h) \mathrm{d} z+p_{m}^{2} \int_{-d}^{0}\left(l_{m} \cos l_{m} z+\alpha \sin l_{m} z\right)^{2} \mathrm{~d} z \\
& =\frac{q_{m}^{2}}{2}\left(c+\frac{\sin 2 l_{m} c}{2 l_{m}}\right)+\frac{p_{m}^{2}}{2}\left(\frac{\left(l_{m}^{2}-\alpha^{2}\right) \sin 2 l_{m} d}{2 l_{m}}+d\left(l_{m}^{2}+\alpha^{2}\right)-2 \alpha \sin ^{2} l_{m} d\right),
\end{aligned}
$$

which, together with (41), determines $p_{m}$.

Now $\phi^{+}$can be expanded in eigenfunctions as

$$
\phi^{+}(x, z)=\sum_{m=0}^{\infty} b_{m} \Psi_{m}(z) \frac{\cosh \lambda_{m}(a-x)}{\cosh \lambda_{m} a} .
$$

Note that this expansion ensures that $\phi^{+}$satisfies (1a), (1b), (1c) and (37).

The vertical eigenfunctions $\Psi_{m}$ satisfy (44). However, since $\mu$ is complex, they do not form an orthonormal set in the classical sense as the corresponding operator is not self-adjoint (see [6] for a detailed discussion in a related context). Moreover, we have no proof that the set is complete.

\subsection{Eigenfunction matching}

Using the expansions (38) and (45), matching the potential and its derivative across $x=0$ respectively gives

$$
\begin{aligned}
\psi_{0}(z)+\sum_{m=0}^{\infty} a_{m} \psi_{m}(z) & =\sum_{m=0}^{\infty} b_{m} \Psi_{m}(z),-h<z<0, \\
-\kappa_{0} \psi_{0}(z)+\sum_{m=0}^{\infty} \kappa_{m} a_{m} \psi_{m}(z) & =-\sum_{m=0}^{\infty} \lambda_{m} b_{m} \Psi_{m}(z) \tanh \lambda_{m} a,-h<z<0 .
\end{aligned}
$$

In general, it is possible to multiply either by $\psi_{n}$ or by $\Psi_{n}$ and integrate over $(-h, 0)$ to obtain an infinite system of equations. But for the finite plate, progress with the residue calculus technique is only possible if we use $\Psi_{n}$. We get

$$
\begin{aligned}
C_{0 n}+\sum_{m=0}^{\infty} a_{m} C_{m n} & =b_{n} \\
-\kappa_{0} C_{0 n}+\sum_{m=0}^{\infty} \kappa_{m} a_{m} C_{m n} & =-\lambda_{n} b_{n} \tanh \lambda_{n} a,
\end{aligned}
$$

$n=0,1, \ldots$, where

$$
C_{m n}=\int_{-h}^{0} \psi_{m}(z) \Psi_{n}(z) \mathrm{d} z .
$$

The integral of the product of the left-hand and right-hand vertical eigenfunctions can be calculated explicitly. From

$$
\psi_{m}^{\prime \prime}(z) \Psi_{n}(z)-\psi_{m}(z) \Psi_{n}^{\prime \prime}(z)=-\left(k_{m}^{2}-l_{n}^{2}\right) \psi_{m}(z) \Psi_{n}(z)
$$


integration from $-h$ to 0 , integration by parts and use of the boundary conditions satisfied by $\psi_{m}$ and $\Psi_{n}$ gives

$$
\begin{aligned}
C_{m n}=\int_{-h}^{0} \psi_{m}(z) \Psi_{n}(z) \mathrm{d} z & =\frac{-1}{k_{m}^{2}-l_{n}^{2}} \int_{-h}^{0} \psi_{m}^{\prime \prime}(z) \Psi_{n}(z)-\psi_{m}(z) \Psi_{n}^{\prime \prime}(z) \mathrm{d} z \\
& =\frac{\psi_{m}^{\prime}(-d)\left(\bar{\Psi}_{n}(-d)-\underline{\Psi}_{n}(-d)\right)}{k_{m}^{2}-l_{n}^{2}} \\
& =\frac{\psi_{m}^{\prime}(-d) \Psi_{n}^{\prime}(-d)}{\mathrm{i} \mu\left(k_{m}^{2}-l_{n}^{2}\right)}
\end{aligned}
$$

Stopping the analysis here and solving system (48) numerically by truncation is the standard eigenfunction matching method used by $[2,1,17]$. The convergence is rather slow, cf. Sect. 5, and further analytical progress is possible: system (48) is also the basis for the residue calculus presented in the next subsection.

\subsection{Residue calculus}

Multiplying (48a) by $\lambda_{n}$, substituting $C_{m n}$ from (51) and making use of $k_{m}^{2}-l_{n}^{2}=$ $\kappa_{m}^{2}-\lambda_{n}^{2}$, (48a) and (48b) become

$$
\begin{aligned}
\lambda_{n} \frac{\psi_{0}^{\prime}(-d) \Psi_{n}^{\prime}(-d)}{\left(\kappa_{0}^{2}-\lambda_{n}^{2}\right)}+\lambda_{n} \sum_{m=0}^{\infty} a_{m} \frac{\psi_{m}^{\prime}(-d) \Psi_{n}^{\prime}(-d)}{\left(\kappa_{m}^{2}-\lambda_{n}^{2}\right)}=\mathrm{i} \mu \lambda_{n} b_{n} \\
-\kappa_{0} \frac{\psi_{0}^{\prime}(-d) \Psi_{n}^{\prime}(-d)}{\left(\kappa_{0}^{2}-\lambda_{n}^{2}\right)}+\sum_{m=0}^{\infty} \kappa_{m} a_{m} \frac{\psi_{m}^{\prime}(-d) \Psi_{n}^{\prime}(-d)}{\left(\kappa_{m}^{2}-\lambda_{n}^{2}\right)}=-\mathrm{i} \mu \lambda_{n} b_{n} \frac{\mathrm{e}^{2 \lambda_{n} a}-1}{\mathrm{e}^{2 \lambda_{n} a}+1} .
\end{aligned}
$$

Multiplying the former equation by $\left(\mathrm{e}^{2 \lambda_{n} a}-1\right)$ and the latter by $\left(\mathrm{e}^{2 \lambda_{n} a}+1\right)$, addition eventually leads to

$$
-\left(\frac{1}{\kappa_{0}+\lambda_{n}}+\frac{\mathrm{e}^{-2 \lambda_{n} a}}{\kappa_{0}-\lambda_{n}}\right)+\sum_{m=0}^{\infty} A_{m}\left(\frac{1}{\kappa_{m}-\lambda_{n}}+\frac{\mathrm{e}^{-2 \lambda_{n} a}}{\kappa_{m}+\lambda_{n}}\right)=0, \quad m=0,1, \ldots,
$$

where

$$
A_{m}=\frac{a_{m} \psi_{m}^{\prime}(-d)}{\psi_{0}^{\prime}(-d)} .
$$

Neglecting the summands involving the exponentials is equivalent to letting $a \rightarrow \infty$ and gives the infinite system for the semi-infinite plate. This is

$$
\sum_{m=0}^{\infty} \frac{A_{m}^{\infty}}{\kappa_{m}-\lambda_{n}}=\frac{1}{\kappa_{0}+\lambda_{n}}, \quad m=0,1, \ldots,
$$

so $R=A_{0}$ and $R^{\infty}=A_{0}^{\infty}$ from (38). We solve (56) as in [19] by considering

$$
I_{m}=\lim _{N \rightarrow \infty} \frac{1}{2 \pi \mathrm{i}} \int_{\mathcal{C}_{N}} \frac{f(z)}{z-\lambda_{m}} \mathrm{~d} z, \quad m=0,1, \ldots,
$$

where $\mathcal{C}_{N}$ is a sequence of circles of radius $(N-1 / 4) \pi$ centred at the origin, say. Also, $f(z)$ has simple poles at $z=-\kappa_{0}, z=\kappa_{n}, n=0,1, \ldots$, and simple zeros at $z=\lambda_{n}$, 
$n=0,1, \ldots$, by assumption and $f(z)=\mathcal{O}\left(z^{-1 / 2}\right)$ as $|z| \rightarrow \infty$ on $\mathcal{C}_{N}$ as $N \rightarrow \infty$. Then, $I_{m}=0$ and, by Cauchy's residue theorem, it follows that

$$
\sum_{n=0}^{\infty} \frac{\operatorname{Res}\left(f ; z=\kappa_{n}\right)}{\kappa_{n}-\lambda_{m}}+\frac{\operatorname{Res}\left(f ; z=-\kappa_{0}\right)}{-\kappa_{0}-\lambda_{m}}=0, \quad m=0,1, \ldots,
$$

so that from (56),

$$
A_{n}^{\infty}=\operatorname{Res}\left(f ; z=\kappa_{n}\right), \quad 1=\operatorname{Res}\left(f ; z=-\kappa_{0}\right), \quad R^{\infty}=\operatorname{Res}\left(f ; z=\kappa_{0}\right) .
$$

Let

$$
f(z)=C g(z), \quad g(z)=\frac{1}{z+\kappa_{0}} \prod_{n=0}^{\infty} \frac{1-z / \lambda_{n}}{1-z / \kappa_{n}} \mathrm{e}^{\chi(z)}
$$

where

$$
\chi(z)=\frac{z}{\pi}(h \log h-c \log c-d \log d),
$$

so that the infinite product converges, or that $\left|\lambda_{n}^{-1}-\kappa_{n}^{-1}\right|=\mathcal{O}\left(n^{-2}\right)$ as $n \rightarrow \infty$. Then, from (59),

$$
\begin{aligned}
R^{\infty} & =C \frac{-\kappa_{0}}{2 \kappa_{0}} \frac{\prod_{n=0}^{\infty}\left(1-\kappa_{0} / \lambda_{n}\right)}{\prod_{n=1}^{\infty}\left(1-\kappa_{0} / \kappa_{n}\right)} \mathrm{e}^{\chi\left(\kappa_{0}\right)}=C \frac{-\left(\lambda_{0}-\kappa_{0}\right)}{2 \lambda_{0}} \prod_{n=1}^{\infty} \frac{\left(1-\kappa_{0} / \lambda_{n}\right)}{\left(1-\kappa_{0} / \kappa_{n}\right)} \mathrm{e}^{\chi\left(\kappa_{0}\right)}, \\
1 & =C \prod_{n=0}^{\infty} \frac{1+\kappa_{0} / \lambda_{n}}{1+\kappa_{0} / \kappa_{n}} \mathrm{e}^{\chi\left(-\kappa_{0}\right)}=C \frac{\lambda_{0}+\kappa_{0}}{2 \lambda_{0}} \prod_{n=1}^{\infty} \frac{1+\kappa_{0} / \lambda_{n}}{1+\kappa_{0} / \kappa_{n}} \mathrm{e}^{-\chi\left(\kappa_{0}\right)} .
\end{aligned}
$$

Solving the latter equation for $C$ and substituting into the former gives

$$
R^{\infty}=\frac{\kappa_{0}-\lambda_{0}}{\kappa_{0}+\lambda_{0}} \prod_{n=1}^{\infty}\left(\frac{1+\mathrm{i} \kappa / \lambda_{n}}{1-\mathrm{i} \kappa / \lambda_{n}}\right)\left(\frac{1-\mathrm{i} \kappa / \kappa_{n}}{1+\mathrm{i} \kappa / \kappa_{n}}\right) \mathrm{e}^{-2 \mathrm{i} \chi(\kappa)}
$$

since $\kappa_{0}=-\mathrm{i} \kappa$ so

$$
R^{\infty}=\frac{\kappa-\mathrm{i} \lambda_{0}}{\kappa+\mathrm{i} \lambda_{0}} \mathrm{e}^{-2 \mathrm{i}(\theta+\chi(\kappa))}, \quad \text { where } \theta=\sum_{n=1}^{\infty}\left(\arctan \left(\kappa / \kappa_{n}\right)-\arctan \left(\kappa / \lambda_{n}\right)\right),
$$

which is the same expression as was obtained by the Wiener-Hopf method using the standard infinite-product decomposition, as in Appendix C. If $\beta=0$,

$$
R^{\infty}=\frac{k-\mathrm{i} l_{0}}{k+\mathrm{i} l_{0}} \mathrm{e}^{-2 \mathrm{i}(\theta+\chi(k))}, \quad \text { where } \theta=\sum_{n=1}^{\infty}\left(\arctan \left(k / k_{n}\right)-\arctan \left(k / l_{n}\right)\right) .
$$

In the finite plate case, given by (54), we let

$$
J_{m}=\lim _{N \rightarrow \infty} \frac{1}{2 \pi \mathrm{i}} \int_{\mathcal{C}_{N}} f(z)\left(\frac{1}{z-\lambda_{m}}+\frac{\mathrm{e}^{-2 \lambda_{m} a}}{z+\lambda_{m}}\right) \mathrm{d} z, \quad m=0,1, \ldots,
$$

and we write

$$
f(z)=C\left(1+\sum_{n=0}^{\infty} \frac{B_{n}}{z-\lambda_{n}}\right) g(z), \quad g(z)=\frac{1}{z+\kappa_{0}} \prod_{n=0}^{\infty} \frac{1-z / \lambda_{n}}{1-z / \kappa_{n}} \mathrm{e}^{\chi(z)}
$$


as before. Provided that $f(z)=\mathcal{O}\left(z^{-1 / 2}\right)$ for $|z| \rightarrow \infty$ on $\mathcal{C}_{N}$ as $N \rightarrow \infty, J_{m}=0$ so

$$
\begin{aligned}
\sum_{n=0}^{\infty} \operatorname{Res}\left(f ; z=\kappa_{n}\right)\left(\frac{1}{\kappa_{n}-\lambda_{m}}\right. & \left.+\frac{\mathrm{e}^{-2 \lambda_{m} a}}{\kappa_{n}+\lambda_{m}}\right)+\operatorname{Res}\left(f ; z=-\kappa_{0}\right)\left(\frac{1}{-\kappa_{0}-\lambda_{m}}+\frac{\mathrm{e}^{-2 \lambda_{m} a}}{-\kappa_{0}+\lambda_{m}}\right) \\
& +f\left(\lambda_{m}\right)+\mathrm{e}^{-2 \lambda_{m} a} f\left(-\lambda_{m}\right)=0, \quad m=0,1, \ldots \quad
\end{aligned}
$$

Note that $\lambda_{m}$ is not automatically a zero of $f$ in this case. In fact, comparing (54) with (69), we require

$$
f\left(\lambda_{m}\right)+\mathrm{e}^{-2 \lambda_{m} a} f\left(-\lambda_{m}\right)=0, \quad m=0,1, \ldots,
$$

as well as

$$
\operatorname{Res}\left(f ; z=\kappa_{n}\right)=A_{n}, \quad \operatorname{Res}\left(f ; z=-\kappa_{0}\right)=1
$$

as before. Now

$$
f\left(-\lambda_{m}\right)=C\left(1-\sum_{n=0}^{\infty} \frac{B_{n}}{\lambda_{m}+\lambda_{n}}\right) \frac{1}{-\lambda_{m}+\kappa_{0}} \prod_{n=0}^{\infty} \frac{1+\lambda_{m} / \lambda_{n}}{1+\lambda_{m} / \kappa_{n}} \mathrm{e}^{-\chi\left(\lambda_{m}\right)}
$$

whilst

$$
f\left(\lambda_{m}\right)=-\frac{C B_{m}}{\lambda_{m}\left(\lambda_{m}+\kappa_{0}\right)} \frac{\prod_{n=0}^{\infty}{ }^{\prime}\left(1-\lambda_{m} / \lambda_{n}\right)}{\prod_{n=0}^{\infty}\left(1-\lambda_{m} / \kappa_{n}\right)} \mathrm{e}^{\chi\left(\lambda_{m}\right)},
$$

where the prime means that the term $n=m$ is missing. So (70) becomes

$$
B_{m}+\sum_{n=0}^{\infty} K_{m n} B_{n}=C_{m}, \quad m=0,1, \ldots
$$

where

$$
\begin{aligned}
C_{m} & =\lambda_{m}\left(\lambda_{m}+\kappa_{0}\right) \mathrm{e}^{-2 \lambda_{m} a} g\left(-\lambda_{m}\right) \mathrm{e}^{-\chi\left(\lambda_{m}\right)} \frac{\prod_{n=0}^{\infty}\left(1-\lambda_{m} / \kappa_{n}\right)}{\prod_{n=0}^{\infty} '\left(1-\lambda_{m} / \lambda_{n}\right)} \\
& =2 \lambda_{m} \frac{\left(\kappa_{0}+\lambda_{m}\right)}{\left(\kappa_{0}-\lambda_{m}\right)} \frac{\left(\kappa_{m}-\lambda_{m}\right)}{\left(\kappa_{m}+\lambda_{m}\right)} \mathrm{e}^{-2\left(\lambda_{m} a+\chi\left(\lambda_{m}\right)\right)} \prod_{n=0}^{\infty} \frac{\left(1-\lambda_{m} / \kappa_{n}\right)}{\left(1+\lambda_{m} / \kappa_{n}\right)} \frac{\left(1+\lambda_{m} / \lambda_{n}\right)}{\left(1-\lambda_{m} / \lambda_{n}\right)}, \\
K_{m n} & =\frac{C_{m}}{\lambda_{m}+\lambda_{n}} .
\end{aligned}
$$

The $l_{2}$ infinite system (74) has a unique solution $B_{n}$ satisfying $\sum_{n=0}^{\infty}\left|B_{n}\right|^{2}<\infty$ if

$$
\sum_{n=0}^{\infty}\left|C_{n}\right|^{2}<\infty, \quad \sum_{m=0}^{\infty} \sum_{n=0}^{\infty}\left|K_{m n}\right|^{2}<1
$$

[see 19, p. 53]. Because of the exponential term in $C_{m}$ and $K_{m n}$, it an be shown that these conditions are satisfied for $\lambda_{0} a$ sufficiently large, i.e. for sufficiently long wavelength or plate. Note that conditions (77) do not yield a useful numerical check whether the requirements are fulfilled. Nevertheless, the truncated version of the second condition gives a sufficient criterion for a given parameter set to be inadmissible. The solution can be written in terms of a convergent Neumann series and a simple iterative solution begins with $B_{n} \sim C_{n}$ etc. Alternatively, (74) can be solved easily by truncation. 
Returning to (71), we have

$$
\operatorname{Res}\left(f ; z=-\kappa_{0}\right)=1=C\left(1-\sum_{n=0}^{\infty} \frac{B_{n}}{\kappa_{0}+\lambda_{n}}\right) \prod_{n=0}^{\infty} \frac{1+\kappa_{0} / \lambda_{n}}{1+\kappa_{0} / \kappa_{n}} \mathrm{e}^{\chi\left(-\kappa_{0}\right)},
$$

which determines $C$. Also, from (71),

$$
A_{0}=R=\operatorname{Res}\left(f ; z=\kappa_{0}\right)=-\frac{C\left(\lambda_{0}-\kappa_{0}\right)}{2 \lambda_{0}}\left(1-\sum_{n=0}^{\infty} \frac{B_{n}}{\lambda_{n}-\kappa_{0}}\right) \prod_{n=1}^{\infty} \frac{1-\kappa_{0} / \lambda_{n}}{1-\kappa_{0} / \kappa_{n}} \mathrm{e}^{\chi\left(\kappa_{0}\right)} .
$$

Eliminating $C$ from (79) using (78), we obtain

$$
R=R^{\infty}\left(1-\sum_{n=0}^{\infty} \frac{B_{n}}{\lambda_{n}-\kappa_{0}}\right) /\left(1-\sum_{n=0}^{\infty} \frac{B_{n}}{\lambda_{n}+\kappa_{0}}\right)
$$

where we have used (64).

Equation (80) provides an accurate expression for $R$ showing the correction from the infinite-plate result, which, since $B_{n}=\mathcal{O}\left(\mathrm{e}^{-\lambda_{n} a}\right)$, describes an infinite sequence of exponentially small contributions travelling back and forth between the ends $x=0$ and $x=a$.

We return to (75) and (68) and estimate $C_{m}$ for large $m$. We write

$$
C_{m}=\frac{\left(\lambda_{m}+\kappa_{0}\right)\left(1-\lambda_{m} / \kappa_{m}\right)}{\left(\kappa_{0}-\lambda_{m}\right)\left(1+\lambda_{m} / \kappa_{m}\right)} 2 \lambda_{m} \mathrm{e}^{-2\left(\lambda_{m} a+\chi\left(\lambda_{m}\right)\right)} P_{m}
$$

where

$$
P_{m}=\prod_{n=0}^{\infty} \frac{\left(1-\lambda_{m} / \kappa_{n}\right)\left(1+\lambda_{m} / \lambda_{n}\right)}{\left(1+\lambda_{m} / \kappa_{n}\right)\left(1-\lambda_{m} / \lambda_{n}\right)}=\prod_{n=0}^{\infty}{ }^{\prime}\left(1+a_{n m}\right),
$$

where $a_{n m}=\mathcal{O}\left(n^{-2}\right)$ as $n \rightarrow \infty$ provided that $\lambda_{n}^{-1}-\kappa_{n}^{-1}=\mathcal{O}\left(n^{-2}\right)$. If so, then $P_{m}$ is convergent and we can rearrange to give

$$
P_{m}=\prod_{n=0}^{\infty} \frac{\left(1-\kappa_{n} / \lambda_{m}\right)\left(1+\lambda_{n} / \lambda_{m}\right)}{\left(1+\kappa_{n} / \lambda_{m}\right)\left(1-\lambda_{n} / \lambda_{m}\right)} \rightarrow 1 \text { as } m \rightarrow \infty .
$$

It follows from (81) and (83) that, provided $\lambda_{n}^{-1}-\kappa_{n}^{-1}=\mathcal{O}\left(n^{-2}\right)$,

$$
C_{m}=\mathcal{O}\left(\frac{\lambda_{m} \mathrm{e}^{-2\left(\lambda_{m} a+\chi\left(\lambda_{m}\right)\right)}}{m^{2}}\right)
$$

and the conditions (77) easily follow [see 19, p. 58-9]. A good approximation to $R$ comes from the first term $B_{0}$ in (80). Now,

$$
B_{0} \sim C_{0}=2 \lambda_{0} \mathrm{e}^{-2\left(\lambda_{0} a+\chi\left(\lambda_{0}\right)\right)} P_{0}, \quad P_{0}=\prod_{n=1}^{\infty}\left(\frac{\kappa_{n}-\lambda_{0}}{\kappa_{n}+\lambda_{0}}\right)\left(\frac{\lambda_{n}+\lambda_{0}}{\lambda_{n}-\lambda_{0}}\right)=\mathrm{e}^{-2 \vartheta},
$$

where

$$
\vartheta=\sum_{n=1}^{\infty}\left(\operatorname{arctanh}\left(\lambda_{0} / \kappa_{n}\right)-\operatorname{arctanh}\left(\lambda_{0} / \lambda_{n}\right)\right)
$$

So

$$
R \sim R^{\infty}\left(1-\frac{B_{0}}{\lambda_{0}-\kappa_{0}}\right) /\left(1-\frac{B_{0}}{\lambda_{0}+\kappa_{0}}\right), \quad B_{0}=2 \lambda_{0} \mathrm{e}^{-2\left(\lambda_{0} a+\chi\left(\lambda_{0}\right)+\vartheta\right)} .
$$


4.4 Fluid extending to infinity in both directions

In the same way, it is possible to solve the problem with a homogeneous Dirichlet condition at $x=a$, i.e. (37) replaced by $\phi=0$ on $x=a$. It is well known that combinations of the reflection coefficients for the symmetric case (homogeneous Neumann condition) and the antisymmetric case (homogeneous Dirichlet condition) give the reflection and transmission coefficients of the corresponding finite structure having twice the length. Denoting the reflection coefficient of the symmetric problem (given by (80)) by $R^{\mathrm{S}}$ and that of the antisymmetric problem by $R^{\mathrm{a}}$, we have the reflection and transmission coefficient

$$
R=\frac{1}{2}\left(R^{\mathrm{s}}+R^{\mathrm{a}}\right), \quad T=\frac{1}{2}\left(R^{\mathrm{S}}-R^{\mathrm{a}}\right)
$$

for a submerged plate occupying $(0,2 a) \times\{-d\}$ in water of infinite extent in both horizontal directions. We briefly outline how the analysis above changes for the antisymmetric case.

In order to accommodate the homogeneous Dirichlet condition at $x=a$, the potential $\phi^{+}$(cf. (45)) is expanded as

$$
\phi^{+, \mathrm{a}}(x, z)=\sum_{m=0}^{\infty} b_{m}^{\mathrm{a}} \Psi_{m}(z) \frac{\sinh \lambda_{m}(a-x)}{\sinh \lambda_{m} a},
$$

where we have added a superscript a to designate the antisymmetric case. This implies that the term $\tanh \lambda_{n} a$ in (48b) needs to be replaced by $\operatorname{coth} \lambda_{n} a$. Equation (54) becomes

$$
-\left(\frac{1}{\kappa_{0}+\lambda_{n}}-\frac{\mathrm{e}^{-2 \lambda_{n} a}}{\kappa_{0}-\lambda_{n}}\right)+\sum_{m=0}^{\infty} A_{m}^{\mathrm{a}}\left(\frac{1}{\kappa_{m}-\lambda_{n}}-\frac{\mathrm{e}^{-2 \lambda_{n} a}}{\kappa_{m}+\lambda_{n}}\right)=0, \quad m=0,1, \ldots,
$$

where

It is found that

$$
A_{m}^{\mathrm{a}}=\frac{a_{m}^{\mathrm{a}} \psi_{m}^{\prime}(-d)}{\psi_{0}^{\prime}(-d)}
$$

$$
R^{\mathrm{a}}=R^{\infty}\left(1-\sum_{n=0}^{\infty} \frac{B_{n}^{\mathrm{a}}}{\lambda_{n}-\kappa_{0}}\right) /\left(1-\sum_{n=0}^{\infty} \frac{B_{n}^{\mathrm{a}}}{\lambda_{n}+\kappa_{0}}\right)
$$

where $B_{n}^{\mathrm{a}}$ is the solution of

$$
B_{m}^{\mathrm{a}}-\sum_{n=0}^{\infty} K_{m n} B_{n}^{\mathrm{a}}=-C_{m}, \quad m=0,1, \ldots
$$

\section{Numerical comparisons}

We present numerical results for some typical parameter sets. Further results can be found in [14].

We begin by comparing $R^{\infty}$ calculated using the expression from the Wiener-Hopf method to the results from simple eigenfunction matching and then compare results for $R$ from the residue calculus technique with eigenfunction matching for a finite plate. It turns out that results of the different methods agree overall but that the convergence 


$\begin{array}{ll}\text { W-H } & 0.0134-0.0539 i \\ \text { EM with } 50 \text { roots } & 0.0130-0.0540 \mathrm{i} \\ \text { EM with } 100 \text { roots } & 0.0132-0.0540 \mathrm{i} \\ \text { EM with } 150 \text { roots } & 0.0132-0.0540 \mathrm{i} \\ \text { EM with 200 roots } & 0.0133-0.0540 \mathrm{i}\end{array}$

Table 1 Reflection coefficient $R^{\infty}$ for $k h=1.2, \sigma=1 / 3, c / h=0.7$ and $\theta_{\text {inc }}=0$.

$\begin{array}{ll}\text { W-H } & 0.2192-0.1755 \mathrm{i} \\ \text { EM with } 50 \text { roots } & 0.2183-0.1766 \mathrm{i} \\ \text { EM with } 100 \text { roots } & 0.2188-0.1761 \mathrm{i} \\ \text { EM with } 150 \text { roots } & 0.2189-0.1759 \mathrm{i} \\ \text { EM with } 200 \text { roots } & 0.2190-0.1758 \mathrm{i}\end{array}$

Table 2 Reflection coefficient $R^{\infty}$ for $k h=0.8, \sigma=1 / 30, c / h=0.9$ and $\theta_{\text {inc }}=0$.

of the eigenfunction matching method is quite slow. Unfortunately, it is impossible to quantitatively compare our results to those of [1] and [17] because only plots of simulation results (rather than numerical values) are given by the authors and slight differences or the slowness of the convergence would not be resolved. We also present some plots of $\left|R^{\infty}\right|$ as a function of $k h$ for several porosities and submergences as such data does not seem to have been given elsewhere and we refer to [1] and [17] for analogous results for the finite plate.

We first compare $R^{\infty}$ calculated using the expression from the Wiener-Hopf approach using the Cauchy integral method $(\mathrm{W}-\mathrm{H})$, i.e. (29) with (30), to the result from simple eigenfunction matching (EM), i.e. (56). The roots of (43) are tedious to calculate and they are found by using their asymptotics to obtain an initial guess, which feeds into a numerical solver based on [20]. Expression (103) is used for the computation of $I(\kappa)$.

We give two generic examples for the convergence of the methods. As a first parameter set, we consider a shorter wave and a plate with a higher porosity submerged at about one third of the water depth. For $k h=1.2, \sigma=1 / 3, c / h=0.7, \theta_{\text {inc }}=0$, the results are given in table 1 . The second parameter set refers to a longer wave and a less porous plate closer to the water surface. For $k h=0.8, \sigma=1 / 30, c / h=0.9, \theta_{\text {inc }}=0$, the computed reflection coefficients are found in table 2.

It can be seen that the results using EM agree with those from the $\mathrm{W}-\mathrm{H}$ approach but the convergence of EM is slow. The behaviour of $\left|R^{\infty}\right|$ for different parameters is shown in figures 1 and 2 .

The fourth decimal place accuracy can be obtained by the semi-infinite-plate residue calculus in the examples above by truncating the series (36) at only $n=9$ and $n=8$, resp. This agreement validates the applicability of the residue calculus method (RC).

We use the residue calculus method for the finite plate (i.e. (80) with (74)) to find $R$ for $a / h=8$ and the other data as in the first example above as well as for $a / h=10$ and the other data as in the second example above. The results are compared to the RC 0th-term approximation (given by (87) and (86)) and the eigenfunction matching method, i.e. (54), for different truncations in tables 3 and 4.

The RC has converged to fourth decimal place accuracy using $n=7$ in the first example (here, $\lambda_{0} a=2.4812-9.5639 \mathrm{i}$ ) and $n=3$ in the second example (here, $\left.\lambda_{0} a=1.8417-23.3324 \mathrm{i}\right)$. For the RC 0th-term approximation this is true for only $n=5$ and $n=3$, resp. 

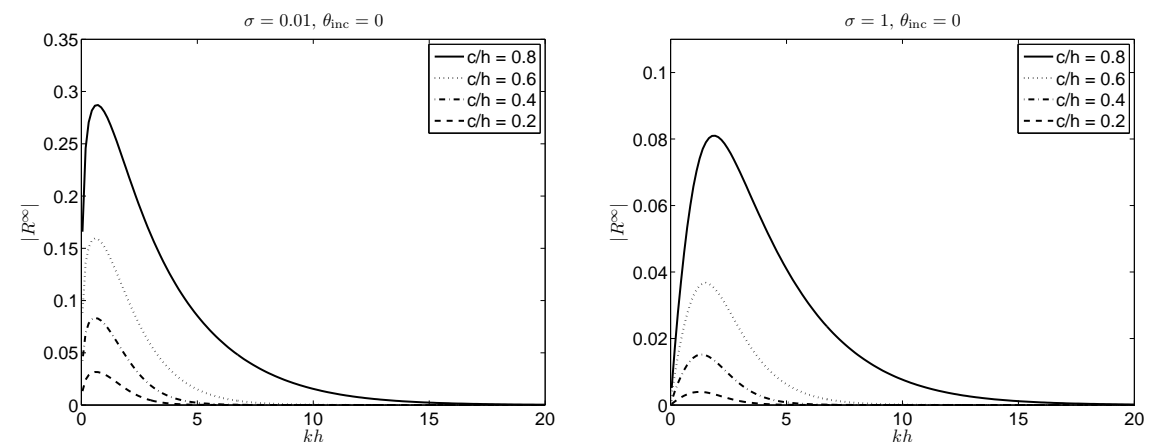

Fig. 1 Reflection coefficient $\left|R^{\infty}\right|$ versus $k h$ for $\sigma=0.01$ (left) and $\sigma=1$ (right), $\theta_{\text {inc }}=0$ and different $c / h$.
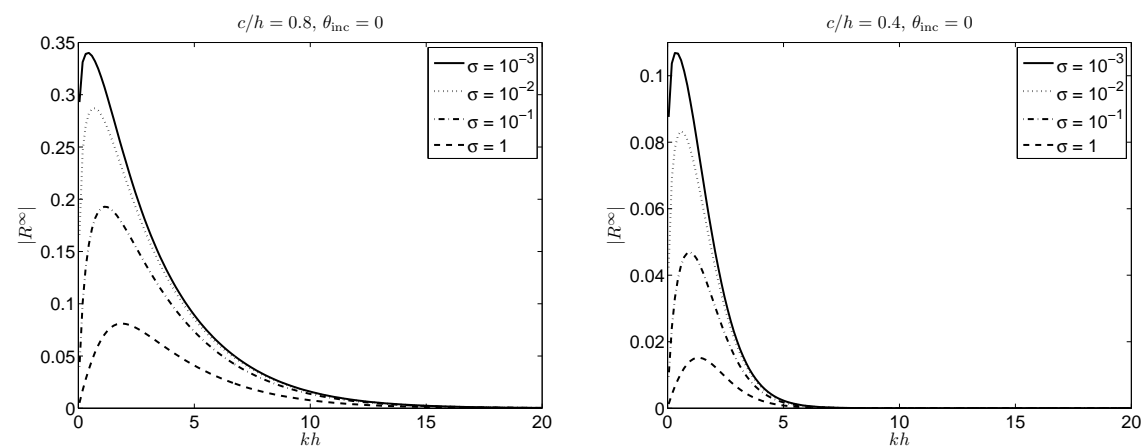

Fig. 2 Reflection coefficient $\left|R^{\infty}\right|$ versus $k h$ for $c / h=0.8$ (left) and $c / h=0.4$ (right), $\theta_{\text {inc }}=0$ and different $\sigma$.

It can be seen that the convergence of the EM is even slower for the finite plate and the RC 0th-term approximation can already yield more accurate results than the EM with 200 roots. In the first example, it even seems to be the case that the EM does not converge to the correct solution but to something close to it (at least when considering only the first few hundred roots), an observation we have made for several parameter sets and which is in agreement with the findings of Porter and Evans [4] for a fixed partially immersed vertical barrier.

The RC proves to be a powerful method for solving such finite-plate problems and, in particular, the 0th-term approximation to the RC method is shown to provide a good approximation to $R$ for a range of parameter sets. However, care should be taken not to apply it in cases for which the conditions (77) are not satisfied, where the full $\mathrm{RC}$ method can appear to converge but differs widely from the zeroth approximation, and where both results differ from the EM results.

\section{Conclusions}

The problem of linear water-wave reflection by a submerged porous plate has been solved for both the semi-infinite plate and the finite plate backed by a solid wall. The 


$\begin{array}{ll}\text { RC } & 0.0201-0.0494 \mathrm{i} \\ \text { RC 0th-term approx. } & 0.0200-0.0496 \mathrm{i} \\ \text { EM with 50 roots } & 0.0206-0.0504 \mathrm{i} \\ \text { EM with 100 roots } & 0.0208-0.0503 \mathrm{i} \\ \text { EM with 150 roots } & 0.0208-0.0503 \mathrm{i} \\ \text { EM with 200 roots } & 0.0209-0.0503 \mathrm{i}\end{array}$

Table 3 Reflection coefficient $R$ for $k h=1.2, \sigma=1 / 3, c / h=0.7, \theta_{\text {inc }}=0$ and $a / h=8$.

$\begin{array}{ll}\text { RC } & 0.2114-0.1764 \mathrm{i} \\ \text { RC 0th-term approx. } & 0.2092-0.1687 \mathrm{i} \\ \text { EM with } 50 \text { roots } & 0.2100-0.1773 \mathrm{i} \\ \text { EM with } 100 \text { roots } & 0.2105-0.1767 \mathrm{i} \\ \text { EM with } 150 \text { roots } & 0.2107-0.1765 \mathrm{i} \\ \text { EM with 200 roots } & 0.2108-0.1764 \mathrm{i}\end{array}$

Table 4 Reflection coefficient $R$ for $k h=0.8, \sigma=1 / 30, c / h=0.9, \theta_{\text {inc }}=0$ and $a / h=10$.

extension to reflection and transmission by a finite plate in a fluid extending to infinity in front and behind the plate have been discussed.

Using the Wiener-Hopf method together with the Cauchy integral method, an easily computable analytic expression for the reflection coefficient $R^{\infty}$ for the semiinfinite plate problem has been derived. This expression does not involve any of the roots of the complicated dispersion relation in the plate region, stemming from the boundary condition across the porous plate, but an easily computable integral with finite integration limits.

For the finite plate backed by a wall, the residue calculus technique has been used to find both a quickly convergent expression for the reflection coefficient $R$ as well as a simple but accurate approximation. The residue calculus technique also gave the same result for the semi-infinite plate as the Wiener-Hopf method together with an infinite-product expansion. All of these approaches require knowledge of the location of the roots of the dispersion relation in the plate region. It turned out that the reflection coefficient $R$ of the finite plate can be written as the product of the semi-infinite plate reflection coefficient $R^{\infty}$ and a correction factor.

The finite-plate problem with a solid wall corresponds to waves being symmetric about the position of the wall. The solution of the related antisymmetric problem, where the homogeneous Neumann condition at the wall is replaced by a homogeneous Dirichlet condition, has been discussed. Both solutions can be combined to give a solution to the problem of a finite submerged porous plate in a fluid extending to infinity in front and behind the plate.

Contrary to simple eigenfunction-matching approaches, all methods considered here specifically account for the singularity of the fluid velocity around the tip of the submerged plate. Numerical computations have shown that all expressions provide powerful methods for solving such problems. In particular, the $n=0$ approximation to the residue calculus result has been shown to provide a good approximation to $R$ for a range of parameter sets, typically similar or even better than the slowly convergent eigenfunction matching method using 200 roots of the dispersion relation.

The methods employed here are not specific to the submerged porous plate and it is likely that the solution approach will work analogously for related problems such as the submerged elastic plate, both semi-infinite and finite, yielding accurate and easily computable expressions for the associated reflection coefficients. 
One minor drawback of the residue calculus solution for the finite plate is the fact that it is only applicable if the plate is long enough compared to the wavelength and that the precise convergence condition does not yield an exhaustive numerical check. The formulation of an exact, easily testable condition in the future would be very useful.

\section{Appendix A Factorisation of $K(s)$ using the Cauchy integral method}

We wish to write $K(s)$ in equation (23) in the form

$$
K(s)=K_{+}(s) K_{-}(s),
$$

where $K_{ \pm}(s)$ is regular and non-zero in $\mathcal{D}_{ \pm}$.

We write $K(s)=G(s) F(s)$, where $G(s)=K^{\mathrm{n}}(s) / K^{\mathrm{o}}(s)$ and $F(s)=K^{\mathrm{o}}(s) / K^{\mathrm{d}}(s)$, where $K^{\mathrm{n}}(s), K^{\mathrm{d}}(s)$ are the numerator and denominator respectively of $K(s)$, and

$$
K^{\mathrm{o}}(s)=\gamma^{2} \sinh \gamma c \sinh \gamma d .
$$

Considering $G(s)$ first, we have

$$
K^{\mathrm{n}}(s)=\gamma \sinh \gamma h-\alpha \cosh \gamma h=\alpha\left(\frac{\gamma^{2}}{k^{2}}-1\right) \prod_{n=1}^{\infty}\left(1+\frac{\gamma^{2}}{k_{n}^{2}}\right)
$$

and

$$
K^{\mathrm{o}}(s)=\gamma \sinh \gamma c \cdot \gamma \sinh \gamma d=\gamma^{4} c d \prod_{n=1}^{\infty}\left(1+\frac{\gamma^{2}}{c_{n}^{2}}\right)\left(1+\frac{\gamma^{2}}{d_{n}^{2}}\right) .
$$

Noting that $h-d-c=0$, it follows that

$$
G_{ \pm}(s)=\frac{\mathrm{e}^{\mp \mathrm{i} \chi(s)} \alpha^{1 / 2} k^{-1}(s \pm \kappa)}{(c d)^{1 / 2}(s \pm i \beta)^{2}} \prod_{n=1}^{\infty} \frac{\left(\kappa_{n} \mp \mathrm{i} s\right) / k_{n}}{\left(c_{n}^{\prime} \mp \mathrm{i} s\right)\left(d_{n}^{\prime} \mp \mathrm{i} s\right) / c_{n} d_{n}}
$$

(see (31) for the definition of $c_{n}^{\prime}, d_{n}^{\prime}$ and so on), where the fact that $k_{n} \sim n \pi / h+\mathcal{O}\left(n^{-1}\right)$ as $n \rightarrow \infty$ ensures the convergence of the infinite product, and the term $\exp ( \pm \mathrm{i} \chi(s))$ is chosen to ensure that $G_{ \pm}(s)$ has algebraic behaviour as $s \rightarrow \infty$ in $\mathcal{D}_{ \pm}$. How this is done is described in [5, pp. 149/150]. The details are messy and only the results are given here. It is found that if

$$
\chi(s)=s \pi^{-1}(h \log h-c \log c-d \log d),
$$

then $G_{+}(s)=\mathcal{O}\left(s^{-1 / 2}\right)$ as $s \rightarrow \infty$ in $\mathcal{D}_{+}$. Note that $G_{+}(s)=-G_{-}(-s)$.

Turning to $F(s)$, we know little about the nature of the roots of the denominator of $F(s)$ other than the fact that they are all complex. In order to avoid evaluating these zeroes, we proceed as follows. We note that $F(s)$ be an even meromorphic function of $s$ which is non-zero in $\mathcal{D}$ and which tends to unity as $|s| \rightarrow \infty$ in $\mathcal{D}$. Then from Cauchy's theorem, we have

$$
\log F_{ \pm}(s)= \pm \frac{1}{2 \pi \mathrm{i}} \int_{\mathcal{C}_{ \pm}} \frac{\log F(t)}{t-s} \mathrm{~d} t
$$

where $\mathcal{C}_{+}\left(\mathcal{C}_{-}\right)$is an infinite contour from $\operatorname{Re} s=-\infty$ to $\operatorname{Re} s=+\infty$ lying in $\mathcal{D}$ and passing below (above) the point $s \in \mathcal{D}$. It follows that $F_{ \pm}(s)$ is regular in $\mathcal{D}_{ \pm}$and $F_{ \pm}(s) \rightarrow 1$ as $s \rightarrow \infty$ in $\mathcal{D}_{ \pm}$.

If $s= \pm \mathrm{i} \nu$, where $\nu \geq \epsilon, \mathcal{C}_{ \pm}$may be shifted onto the real axis and we find that

$$
F_{ \pm}( \pm \mathrm{i} \nu)=\exp \left(\frac{1}{\pi} \int_{0}^{\infty} \frac{\log F(\nu t)}{t^{2}+1} \mathrm{~d} t\right) .
$$

If $s$ is real we can still deform the contour onto the real axis provided we indent above or below the point $s$. We find that

$$
F_{ \pm}(s)=(F(s))^{\frac{1}{2}} \exp (\mp \mathrm{i} I(s))
$$


where

$$
I(s)=\frac{1}{\pi} \int_{0}^{\infty} \frac{\log (F(s t))}{t^{2}-1} \mathrm{~d} t=\frac{1}{\pi} \int_{0}^{1} \frac{\log (F(s / t) / F(s t))}{1-t^{2}} \mathrm{~d} t
$$

after splitting the integration up and writing $1 / t$ for $t$ in the infinite integral. Note that the integrand is well-behaved at $t=1$. Finally we note that $F_{+}(s)=F_{-}(-s)$.

In summary then, we have

$$
K_{ \pm}(s)=G_{ \pm}(s) F_{ \pm}(s)
$$

where $G_{ \pm}(s)$ is given by (98), and $F_{ \pm}(s)$ by (101), (102) and (103), $K_{ \pm}(s)=O\left(s^{-1 / 2}\right)$ as $s \rightarrow \infty$ in $\mathcal{D}_{ \pm}$, and $K_{+}(s)=-K_{-}(-s)$.

\section{Appendix B Simplification of $R$}

In order to obtain (29) from (28), we make use of the fact that $K_{+}(s)=-K_{-}(-s)$ and $K_{+}(s)=F_{+}(s) G_{+}(s)$, where $F_{+}$and $G_{+}$are given by (102) and (98), respectively. Use of the identities

$$
\begin{gathered}
\frac{2 k h+\sinh 2 k h}{4 \sinh k h}=\prod_{n=1}^{\infty}\left(1+\frac{k^{2}}{k_{n}^{2}}\right)=\prod_{n=1}^{\infty}\left(\frac{\kappa_{n}^{2}+\kappa^{2}}{k_{n}^{2}}\right), \\
\frac{\sinh k c}{k c}=\prod_{n=1}^{\infty}\left(1+\frac{k^{2}}{c_{n}^{2}}\right)=\prod_{n=1}^{\infty}\left(\frac{c_{n}^{\prime 2}+\kappa^{2}}{c_{n}^{2}}\right), \\
\frac{\sinh k d}{k d}=\prod_{n=1}^{\infty}\left(1+\frac{k^{2}}{d_{n}^{2}}\right)=\prod_{n=1}^{\infty}\left(\frac{d_{n}^{\prime 2}+\kappa^{2}}{d_{n}^{2}}\right),
\end{gathered}
$$

and noting that $F(\kappa)=-\sinh k d \cosh k h / \sinh k c$ and $(\kappa+i \beta)^{2}=k^{2} \exp (2 \mathrm{i} \arctan \beta / \kappa)$ leads to $(29)$.

\section{Appendix C Factorisation of $K(s)$ using an infinite-product expansion}

The Wiener-Hopf splitting function $K(s)$ can also be factorised using a standard infiniteproduct expansion, which leads to the same representations of $R^{\infty}$ as in (36) and as that obtained by the semi-infinite-plate residue calculus (65).

We have

$$
\gamma h \sinh \gamma h-\alpha h \cosh \gamma h=\alpha h\left(\frac{\gamma^{2}}{k^{2}}-1\right) \prod_{n=1}^{\infty}\left(1+\frac{\gamma^{2}}{k_{n}^{2}}\right) .
$$

So in an obvious notation,

$$
(\gamma h \sinh \gamma h-\alpha h \cosh \gamma h)_{ \pm}=(\alpha h)^{1 / 2} k^{-1}(s \pm \kappa) \prod_{n=1}^{\infty}\left[\left(1+\frac{\beta^{2}}{k_{n}^{2}}\right)^{1 / 2} \mp \frac{\mathrm{i} s}{k_{n}}\right] \mathrm{e}^{ \pm \mathrm{i} s h / n \pi} .
$$

The denominator $K^{\mathrm{d}}(s)$ of $K(s)$ has zeros $\gamma=\mathrm{i} l_{n}$, which are complex (possibly double) but are known, see equation (43). Then,

$$
K^{\mathrm{d}}(s)=\mathrm{i} \mu \alpha \prod_{n=0}^{\infty}\left(1+\frac{\gamma^{2}}{l_{n}^{2}}\right)
$$

and

$$
K_{ \pm}^{\mathrm{d}}(s)=(\mathrm{i} \mu \alpha)^{1 / 2} \prod_{n=0}^{\infty}\left[\left(1+\frac{\beta^{2}}{l_{n}^{2}}\right)^{1 / 2} \mp \frac{\mathrm{i} s}{l_{n}}\right] \mathrm{e}^{ \pm \mathrm{i} s(c+d) / n \pi} .
$$

It follows that $K_{-}(-s)=K_{+}(s)$ so from $(28)$,

$$
R^{\infty}=\frac{k^{3} \sinh ^{2} k c K_{+}(\kappa)^{2}}{\kappa^{2}(2 k h+\sinh 2 k h)},
$$


where

$$
K_{+}(\kappa)=\frac{\alpha^{1 / 2} k^{-1} 2 \kappa}{(\mathrm{i} \mu \alpha)^{1 / 2}} \frac{\prod_{n=1}^{\infty}\left[\left(1+\frac{\beta^{2}}{k_{n}^{2}}\right)^{1 / 2}-\frac{\mathrm{i} \kappa}{k_{n}}\right]}{\prod_{n=0}^{\infty}\left[\left(1+\frac{\beta^{2}}{l_{n}^{2}}\right)^{1 / 2}-\frac{\mathrm{i} \kappa}{l_{n}}\right]} \mathrm{e}^{-\mathrm{i} \chi(\kappa)}
$$

where $\chi(s)$ is chosen as

$$
\chi(s)=\frac{s}{\pi}(h \log h-c \log c-d \log d)
$$

in order to ensure the convergence of the infinite product. Making use of the relations (105) and

$$
\prod_{n=0}^{\infty}\left(1+\frac{k^{2}}{l_{n}^{2}}\right)=\frac{-k}{\mathrm{i} \mu} \frac{\sinh ^{2} k c}{\sinh k h}
$$

we find that

$$
\begin{aligned}
& K_{+}(\kappa)^{2}=\frac{k^{-2} 4 \kappa^{2}}{\mathrm{i} \mu} \frac{\prod_{n=1}^{\infty}\left[\left(1+\frac{\beta^{2}-\kappa^{2}}{k_{n}^{2}}\right)-2\left(1+\frac{\beta^{2}}{k_{n}^{2}}\right)^{1 / 2} \frac{\mathrm{i} \kappa}{k_{n}}\right]}{\prod_{n=0}^{\infty}\left[\left(1+\frac{\beta^{2}-\kappa^{2}}{l_{n}^{2}}\right)-2\left(1+\frac{\beta^{2}}{l_{n}^{2}}\right)^{1 / 2} \frac{\mathrm{i} \kappa}{l_{n}}\right]} \mathrm{e}^{-2 \mathrm{i} \chi(\kappa)} \\
& =\frac{k^{-2} 4 \kappa^{2}}{\mathrm{i} \mu} \frac{\frac{2 k h+\sinh 2 k h}{4 \sinh k h} \prod_{n=1}^{\infty}\left[\left(\frac{\kappa_{n}^{2}-\kappa^{2}}{\kappa_{n}^{2}+\kappa^{2}}\right)-2 \frac{\mathrm{i} \kappa \kappa_{n}}{\kappa_{n}^{2}+\kappa^{2}}\right]}{\frac{-k}{\mathrm{i} \mu} \frac{\sinh ^{2} k c}{\sinh k h} \prod_{n=0}^{\infty}\left[\left(\frac{\lambda_{n}^{2}-\kappa^{2}}{\lambda_{n}^{2}+\kappa^{2}}\right)-2 \frac{\mathrm{i} \kappa \lambda_{n}}{\lambda_{n}^{2}+\kappa^{2}}\right]} \mathrm{e}^{-2 \mathrm{i} \chi(\kappa)} \\
& =-\frac{\kappa^{2}(2 k h+\sinh 2 k h)}{k^{3} \sinh ^{2} k c} \frac{\prod_{n=1}^{\infty}\left[\frac{\kappa_{n}-\mathrm{i} \kappa}{\kappa_{n}+\mathrm{i} \kappa}\right]}{\prod_{n=0}^{\infty}\left[\frac{\lambda_{n}-\mathrm{i} \kappa}{\lambda_{n}+\mathrm{i} \kappa}\right]} \mathrm{e}^{-2 \mathrm{i} \chi(\kappa)}
\end{aligned}
$$

so

$$
R^{\infty}=-\mathrm{e}^{-2 \mathrm{i} \chi(\kappa)} \prod_{n=0}^{\infty} \frac{\lambda_{n}+\mathrm{i} \kappa}{\lambda_{n}-\mathrm{i} \kappa} \cdot \prod_{n=1}^{\infty} \frac{\kappa_{n}-\mathrm{i} \kappa}{\kappa_{n}+\mathrm{i} \kappa} .
$$

Hence, we recover the representation obtained by the residue calculus technique (65) as well as that given in (36).

\section{Appendix D The special case of a dock $(\sigma=0)$}

The special case $\sigma=0$ has a particularly simple form for $|R|$, first derived by [7]. Now,

$$
K(s)=\frac{\gamma \sinh \gamma h-\alpha \cosh \gamma h}{\gamma \sinh \gamma c(\gamma \sinh \gamma d-\alpha \cosh \gamma d)}
$$

and the zeros of the denominator are all either real of pure imaginary. In an abuse of notation, let $\gamma \sinh \gamma d-\alpha \cosh \gamma d$ have zeros $\pm \mathrm{i} l_{n}, n=0,1, \ldots$, with $l_{0}=-\mathrm{i} l$ and $\lambda=\left(l^{2}-\beta^{2}\right)^{1 / 2}$. Then,

$$
(\gamma \sinh \gamma d-\alpha \cosh \gamma d)_{ \pm}=(\alpha d)^{1 / 2} l^{-1}(s \pm \lambda) \prod_{n=1}^{\infty}\left[\left(1+\frac{\beta^{2}}{l_{n}^{2}}\right)^{1 / 2} \mp \frac{\mathrm{i} s}{l_{n}}\right] \mathrm{e}^{ \pm \mathrm{i} s d / n \pi}
$$

and

$$
(\gamma c \sinh \gamma c)_{ \pm}=c(s \pm \mathrm{i} \beta) \prod_{n=1}^{\infty}\left[\left(1+\frac{\beta^{2}}{p_{n}^{2}}\right)^{1 / 2} \mp \frac{\mathrm{i} s}{p_{n}}\right] \mathrm{e}^{ \pm \mathrm{i} s c / n \pi}
$$

where $p_{n}=n \pi / c$, see [5, p. 144-146] for details.

We find eventually that

$$
\left|K_{+}(\kappa)\right|=\frac{4 c^{-1} \kappa^{2} l^{2}}{(\kappa+\lambda)^{2} k^{4}} \prod_{n=1}^{\infty} \frac{1+k^{2} / k_{n}^{2}}{\left(1+k^{2} / p_{n}^{2}\right)\left(1+k^{2} / l_{n}^{2}\right)} .
$$


We make use of

$$
\prod_{n=1}^{\infty}\left(1+\frac{k^{2}}{l_{n}^{2}}\right)=\frac{l^{2} \sinh k c}{\left(\lambda^{2}-\kappa^{2}\right) \sinh k h}, \quad \prod_{n=1}^{\infty}\left(1+\frac{k^{2}}{p_{n}^{2}}\right)=\frac{\sinh k c}{k c}
$$

and find, eventually, that

$$
|R|=\left|\frac{\lambda-\kappa}{\lambda+\kappa}\right|
$$

This provides a check on the results for small non-zero $\sigma$.

\section{References}

1. J. Wu, Z. Wan, and Y. Fang. Wave reflection by a vertical wall with a horizontal submerged porous plate. Ocean Engng, 25(9):767-779, 1998.

2. A. T. Chwang and J. Wu. Wave scattering by submerged porous disk. J. Engng Mech., 120(12):2575-2587, 1994

3. M. Hassan, M. H. Meylan, and M. A. Peter. Water-wave scattering by submerged elastic plates. Q. J. Mech. Appl. Math., 62(3):321-344, 2009.

4. R. Porter and D. V. Evans. Complementary approximations to wave scattering by vertical barriers. J. Fluid Mech., 294:155-180, 1995.

5. C. M. Linton and P. McIver. Handbook of Mathematical Techniques for Wave / Structure Interactions. Chapman \& Hall /CRC, 2001.

6. P. McIver. The dispersion relation and eigenfunction expansions for water waves in a porous structure. J. Engng Math., 34:319-334, 1998.

7. A. E. Heins. Water waves over a channel of finite depth with a submerged plane barrier. Can. J. Math., 2:210-222, 1950.

8. C. M. Linton and D. V Evans. Trapped modes above a submerged horizontal plate. Q. J. Mech. Appl. Math., 44(3):487-506, 1991.

9. D. V. Evans and T. V. Davies. Wave-ice interaction. Technical Report 1313, Davidson Laboratory, Stevens Institute of Technology, New Jersey, 1968.

10. N. J. Balmforth and R. V. Craster. Ocean waves and ice sheets. J. Fluid Mech., 395:89124, 1999.

11. L. A. Tkacheva. The diffraction of surface waves by a floating elastic plate at oblique incidence. J. Appl. Math. Mech., 68(3):425-436, 2004.

12. H. Chung and C. Fox. Calculation of wave-ice interaction using the Wiener-Hopf technique. New Zealand J. Math., 31:1-18, 2002.

13. C. M. Linton and H. Chung. Reflection and transmission at the ocean/sea-ice boundary. Wave Motion, 38(1):43-52, 2003.

14. D. V. Evans and M. A. Peter. Reflection of water waves by a submerged horizontal porous plate. In A. Korobkin and P. Plotnikov, editors, Proc. 24th Int. Workshop on Water Waves and Floating Bodies, Zelenogorsk, Russia, pages 82-85, 2009.

15. A. T. Chwang. A porous-wavemaker theory. J. Fluid. Mech., 132:395-406, 1983.

16. I. S. Gradshteyn, I. M. Ryzhik, A. Jeffrey, and D. Zwillinger, editors. Table of Integrals, Series, and Products. Academic Press, 6 edition, 2000.

17. I. H. Cho and M. H. Kim. Development of wave absorbing system using an inclined porous plate. In H. S. Choi and Y. Kim, editors, Proc. 23rd Int. Workshop on Water Waves and Floating Bodies, Jeju, Korea, pages 25-28, 2008.

18. R. Mittra and S. W. Lee. Analytical techniques in the theory of guided waves. Macmillan, 1971.

19. D. V. Evans. Trapped acoustic modes. IMA J. Appl. Math., 49:45-60, 1992

20. M. H. Meylan and L. Gross. A parallel algorithm to find the zeros of a complex analytic function. ANZIAM J., 44(E):E216-E234, 2003. 